\title{
Networking
}

\section{The need for wider public understanding of health care research}

Brian Buckley Department of General Practice, National University of Ireland, Galway, Ireland

\begin{abstract}
In recent years several factors have affected the public's relationship with health care research: increased data protection legislation and the resultant consent requirements; access to unforeseen levels of both information and misinformation through mass media; and a growing culture of personal choice which may have eroded the perceived importance of activities whose benefits are societal rather than personal. This article considers these factors and their implications and highlights the need for health care researchers to engage more effectively with the public in order to ensure its continued support.
\end{abstract}

Key words: consent; health care research; public engagement

Received: August 2007; accepted: October 2007

\section{Introduction}

Health care researchers enjoy a multifaceted relationship with the public, who are stakeholders in their work in more ways than one. As potential patients, the public are the intended beneficiaries of health care research. As taxpayers they pay for publicly funded research. As consumers of health technologies - whether in a publicly or a privately funded health care system - they ultimately pay for industry-funded research too, albeit less directly.

The public's involvement with health care research does not stop at issues of investment and return. With an astonishing degree of optimism, researchers ask not only that the public pay for their work, but also that, on occasions, they should trust their motives, skills and judgements enough to take part in it. Participation may mean consenting to test therapies, having their health care delivered according to a protocol designed by an unseen researcher rather than their clinician, or

Address for correspondence: Brian Buckley, Department of General Practice, National University of Ireland, Galway, Ireland. Email: bsbuckley@iol.ie allowing researchers access to personal information about their lives and health care.

A great many people have participated in research and thereby contributed to the development of the services from which society as a whole has benefited. However, several recent developments mean that those concerned with health care research must reconsider what they must do to ensure the public's continued support and cooperation. First, the privacy and confidentiality of individuals are more robustly protected than ever before, limiting the ability of health care researchers to secure patients' participation or personal data for research purposes (Al-Shahi et al., 2005; Buckley et al., 2007a). Second, the expansion of broadcast and the Internet media means that the public has access to more information on any topic than was previously imaginable - some of it reliable, some less so. Third, an increasing emphasis has been placed on personal choice in accessing everything from housing and school places through to health care. Wilby (2007) suggests that this focus on the individual's right to choice has diminished public understanding of the obligations that 
public services have to others in the society. It may also have reduced expectations that individuals should participate in activities such as research whose purpose and benefit may be societal rather than personal.

\section{Increased protection of individuals' privacy}

In recent years, data protection laws and more conservative medico-ethical guidelines have placed increasing restrictions on how health care research can gather information from patients. For example, there now exists a broad disapproval of accessing anything but the most rigorously anonymised data for research purposes without seeking each patient's prior consent. In contrast (although it seems unlikely that many of the public understand the distinction), quite different regulations govern access to personal information for the purposes of clinical or financial audit (DoH, 2005).

Concerns have been expressed that while patient privacy and confidentiality must be maintained, there is a danger that such constraints may become too strict - that in order to prevent any harm to individuals, research may be prevented which offers very large benefits to society, creating a different ethical dilemma (Regidor, 2004; Walley, 2006). Recent reports have highlighted the implications for observational research of 'consent bias' (the loss to research of non-consenters), demonstrating that clinically important prognostic variables may be associated with consent preferences. As a result, the effects of treatments may be over-estimated or under-estimated, the generalisability of such research diminished, and much epidemiology and health services research rendered too biased to guide practice (Al-Shahi et al., 2005; Buckley et al., 2007a).

Because of these concerns, health care researchers need to consider how the chances of securing consent can be maximised. In future it may be possible to develop different patient consent requirements for different types of research, but it seems unlikely that restrictions on access to patients' medical information for research purposes will be amended in the short term. As a result, the many studies that are dependent upon these data will depend upon securing consent for the foreseeable future. Recruitment difficulties can also affect trial-based research and in the past the UK has fallen behind many other countries in its ability to recruit the numbers needed for trials (Smith, 2000). One aim of the UK Clinical Research Network is to 'Raise the public profile of clinical research and the health benefits of participating' ( $\mathrm{DoH}, 2006)$. If this initiative is successful, participation in trials may improve.

\section{An informed public?}

The public is now better informed than ever before, but it is probably true to say that they are also more effectively misinformed. On television, the Internet and in the print media it is commonplace for opinion and commentary to be presented as fact; conflicts of interest and hidden agendas are not often apparent to the user; and in the quest for sales or ratings 'a good story' will always trump dull fact. As a result, like many topics, health care research remains poorly understood, and while this remains the case it will be difficult to cultivate support and participation or to counter the misrepresentation of research evidence or the public relations effects of tragedies such as the TGN1412 trial (Goodyear, 2006).

It is not easy for the public to find out about health care research: why it is necessary, how it is prioritised, funded and conducted, and how its findings are used. Although a great deal of information is in the public domain, health care research is a complex and specialised activity which is not easily understood by the outsider. Regarding the use of personal medical information for research purposes, the UK Medical Research Council notes that 'many people will not know how information about them might be used'. This poor public understanding of the research process and what participation might mean has been confirmed in a number of studies (Robling et al., 2004; Williams et al., 2007). The benefit of adequately explained research aims and methods is highlighted by an Irish survey of public attitudes towards retention of tissue samples for research purposes. Of those who said that, if asked, they would allow samples to be stored for future research, initially $50 \%$ agreed 
that such samples could be linked to their personal medical records. However, this increased to $89 \%$ when the potential benefits of linking samples with records was explained to them (Cousins et al., 2005).

Much is made of the importance of procuring patients' 'informed' consent for participation in research. Yet, despite the provision of information leaflets and the opportunity to ask further questions, there is no certainty that those approached to take part in research understand what is involved. A study which contacted people who did not consent to participation in an earlier survey found that the vast majority declined because they had misunderstood what the research required of them or why they had been contacted (Williams et al., 2007). Another factor which may impact on the effectiveness of seeking consent for participation is that this request often comes at a time of ill health. Saying 'no' may be the easiest position to adopt at these times of stress.

It seems likely that if people were better informed, they might be more likely to participate. Improved awareness and understanding of research prior to any invitation to participate in a study may create a better 'baseline predisposition' to participate, so that should such an invitation occur, consent and participation may be more likely. In addition, rare accidents, bad research and bad reporting of research would be more widely recognised for what they are.

Several initiatives may contribute to increased support and understanding of health care research. Social marketing has potential to encourage wider social movement for better health and may also influence attitudes to participation in health research (NCC, 2006). Organisations such as INVOLVE and the James Lind Alliance are working to promote the involvement of patients and carers in the conduct and prioritisation of research (Buckley et al., 2007b).

\section{Promoting research participation as a way of helping others}

In the face of an increasing emphasis on personal choice and a diminishing regard for activities whose benefits are societal rather than personal, public awareness of the benefits of health care research needs to be raised. It is interesting to consider what parallels can be drawn with organ donation. Although insufficient numbers have actually signed donor registers and the shortage of organs persists as a result, support for the principle is undoubtedly widespread (BMA, 2005). It may be useful to contemplate whether the selflessness and altruism which underpins the promotion of organ donation might not similarly be achievable for health services research.

Consent to participation in research ought to be promoted as an opportunity to improve health care and help others. The involvement of the popular media would be valuable in such work. Easily read leaflets should be made widely available in health care settings and elsewhere. The public needs to be more informed about the principles that underpin clinical studies and what participation typically involves. They need reassurance about how information about their own health may be used and how their privacy will be protected. And they need to know how the results of research are used to expand the evidence base, enabling clinicians to make better decisions about their health care and the effective use of resources.

\section{Acknowledgements}

Thanks are due for help in the preparation of this paper to Rustam Al-Shahi Salman, MRC Clinician Scientist and Honorary Consultant Neurologist, Western General Hospital, Edinburgh and Anne MacFarlane, Lecturer in Primary Care, National University of Ireland, Galway.

\section{Conflicts of interest}

None.

\section{References}

Al-Shahi, R., Vousden, C. and Warlow, C. 2005: Bias from requiring explicit consent from all participants in observational research: prospective, population based study. BMJ 331, 942-47.

BMA. 2005: Organ donation - presumed consent for organ donation. London: British Medical Association.

Buckley, B., Murphy, A.W., Byrne, M. and Glynn, L.G. 2007a: Selection bias resulting from the requirement for prior consent in observational research: a community cohort of people with ischaemic heart disease. Heart 93, 1116-120.

Primary Health Care Research \& Development 2008; 9: 3-6 


\section{Brian Buckley}

Buckley, B., Grant, A.M., Firkins, L., Greene, A.C. and Frankau, J. 2007b: Working together to identify research questions. Continence 1, 76-81.

Cousins, G., McGee, H., Ring, L., Conroy, R., Kay, E., Croke, D. and Tomkin, D. 2005: Public perceptions of biomedical research: a survey of the general population in Ireland. Dublin: Health Research Board.

DoH. 2005: Confidentiality and disclosure of information: General Medical Services (GMS), Personal Medical Services (PMS), and Alternative Provider Medical Services (APMS) code of practice. London: Department of Health.

DoH. 2006: Best research for best health. A new national health research strategy. London: Research and Development Directorate, Department of Health.

Goodyear, M. 2006: Learning from the TGN1412 trial. BMJ 332, 677-78.

NCC. 2006: It's our health! Realising the potential of effective social marketing. London: Research and Development Directorate, Department of Health.

Regidor, E. 2004: The use of personal data from medical records and biological materials: ethical perspectives and the basis for legal restrictions in health research. Social Science and Medicine 59, 1975-84.

Robling, M.R., Hood, K., Houston, H., Pill, R., Fay, J. and Evans, H.M. 2004: Public attitudes towards the use of primary care patient record data in medical research without consent: a qualitative study. Journal of Medical Ethics 30, 104-09.

Smith, R. 2000: UK is losing market share in pharmaceutical research. BMJ 321, 1041.

Walley, T. 2006: Using personal health information in medical research. BMJ 332, 130-31.

Wilby, P. 2007: The grousers, the ignorant, the selfish - Peter Wilby argues that fairness must rule over choice. New Statesman, 9 July 2007, p. 18.

Williams, B., Irvine, L., McGinnis, A.R., McMurdo, M.E.T. and Crombie, I.K. 2007: When "no" might not quite mean "no"; the importance of informed and meaningful nonconsent: results from a survey of individuals refusing participation in a health-related research project. $B M C$ Health Services Research 7, 59. 